\title{
ANÁLISIS DE LAS PROPUESTAS DE LOS ACTORES SOCIALES EN LA RECUPERACIÓN AMBIENTAL DE LA BAHÍA DE PORTMÁN (REGIÓN DE MURCIA). NUEVAS PERSPECTIVAS PARA UN DESARROLLO SOSTENIBLE
}

\author{
Isabel Banos-González* \\ Universidad de Fribourg (Suiza) \\ Pedro Baños Páez,** \\ Universidad de Murcia \\ Pedro Pérez-Cutillas***
}

Centro de Edafología y Biología Aplicada del Segura

Consejo Superior de Investigaciones Científicas (CEBAS-CSIC)

Miguel Ángel Esteve Selma****

Universidad de Murcia

\section{RESUMEN}

La bahía de Portmán representa uno de los casos de contaminación más importantes en el Mediterráneo. En este trabajo se analizan las posiciones de los diferentes actores sociales, así como las principales fortalezas-debilidades y oportunidades-amenazas que existen en las posibilidades de un planteamiento estratégico de regeneración de la bahía y de la Sierra Minera. Desde una perspectiva socio-ecológica, los análisis realizados ponen de manifiesto las potencialidades para el desarrollo de un turismo ambiental y cultural de este entorno. Sin embargo, las propias debilidades y amenazas podrían superar esas potencialidades si no se

Recibido: 2 de abril de 2016

Devuelto para su revisión: 12 de diciembre de 2016

Aceptado: 20 de febrero de 2017

* Department of Geosciences. University of Fribourg 4. Chemin du Musée. CH-1700 FRIBOURG (Switzerland). E-mail: isabel.banosgonzalez@unifr.ch

** Departamento de Sociología y Trabajo Social. Facultad de Economía y Empresa. Universidad de Murcia. 30100 Espinardo. MURCIA (España).E-mail: pbanos@um.es

*** Grupo de Erosión y Conservación de Suelos. Universidad de Murcia. 30100 Espinardo MURCIA (España).E-mail: perezcutillas@cebas.csic.es

**** Departamento de Ecología e Hidrología. Facultad de Biología.Universidad de Murcia. 30100 Espinardo MURCIA (España).E mail: maesteve@um.es 
lleva a cabo una gestión cuidadosa y una apuesta clara por un modelo de desarrollo sensible en extremo con el patrimonio natural, paisajístico y cultural. Un modelo de turismo ligado a la restauración ambiental y al consenso con los agentes sociales locales debe constituir un elemento básico para la adaptación del territorio como producto turístico, y así debe reflejarse en las futuras decisiones.

Palabras clave: turismo sostenible, actores sociales, DAFO, restauración ambiental.

\section{Analysis of the proposals of the social actors in the environmental recovery of the Portmán Bay (Murcia). New perspectives for sustainable development}

\section{ABSTRACT}

The Portmán bay is one of the most important cases of pollution in the Mediterranean. In this work the standpoints of the different social actors are analyzed, as well as the main strengths-weaknesses and opportunities-threatens for the strategic planning of the restoration of the bay and the Mining Mountain Range. From a socio-ecological perspective, these analyses show its potential to promote an environmental and cultural tourist development in this area. Nevertheless, the own weaknesses and threats might overcome this potential if a careful management and a clear commitment to a highly sensitive development model with the natural, landscape and cultural heritage are not performed. A tourist model linked to environmental restoration and the consensus of the local stakeholders should constitute a basic element for the adaptation of the territory as a tourist product, and this should be reflected in future decisions.

Keywords: sustainable tourism, social agents, SWOT, environmental restoration.

\section{INTRODUCCIÓN}

La bahía de Portmán, situada a mitad de camino entre las localidades de Cartagena y Cabo de Palos (SE de la Península Ibérica), alberga el escenario de uno de los mayores desastres ambientales del litoral mediterráneo (Martínez-Frias, 1997): la colmatación de la bahía provocada por el vertido de más de 60 millones de toneladas de estériles de minería al mar durante más de treinta años (Banos-González y Baños Páez, 2013).

Importantes restos arqueológicos como los campamentos mineros en La Paloma, el Cabezo del Pino, la Rambla de la Aboltada y la villa romana de Huerta del Paturro (siglos I a IV ddc) muestran que, desde la antigüedad, la Sierra Minera de CartagenaLa Unión ha venido siendo explotada para la obtención de minerales como plata, galena, blenda, cerusita, casiterita y diversos óxidos de hierro. A finales del siglo XIX, Charles Ledoux, directivo de la empresa francesa Société Houillère et Metallurgique de Belmez, constituye la sociedad Minero Metalúrgica de Peñarroya, que se instaló definitivamente en esta Sierra Minera en la segunda mitad del siglo XX, fecha en la que se inicia la verdadera transformación del paisaje natural del entorno de la bahía (Baños Páez, 2012). 
El lavadero de Peñarroya utilizaba agua del mar para la concentración de mineral mediante un proceso de flotación diferencial, llegando a alcanzar una capacidad de procesamiento de hasta $10.000 \mathrm{t}_{\text {día }}{ }^{-1}$ de mineral. Los estériles del proceso, vertidos directamente a la bahía entre 1957 y 1990, ocuparon el área en un radio de varios kilómetros (Benedicto et al., 2013). Tras el cese de los vertidos, más de un $80 \%$ de la bahía de Portmán se encuentra colmatada, lo que equivale a unas 75 ha. que previamente habían estado ocupadas por el mar y cerca de cien kilómetros cuadrados de los fondos marinos de la plataforma continental frente a Portmán (Benedicto et al., 2008). La huella en el paisaje de la Sierra se extiende en unos $40 \mathrm{~km}^{2}$. Los depósitos de estériles de antiguos lavaderos de mineral (los pantanos, en el argot minero de la zona) ocupan unas 270 ha. y las terreras de desescombro de las canteras, que suponen unos 300 millones de toneladas de tierra simplemente apilada, se encuentran distribuidos en los alrededores de las poblaciones de La Unión, Portmán, El Llano del Beal, etc. (Banos-González y Baños Páez, 2013).

Los acontecimientos más recientes en la recuperación de la bahía de Portmán y su entorno se inician el 11 de febrero de 2011, cuando la Secretaria de Estado de Cambio Climático en la reunión de la Comisión de Seguimiento de Portmán anuncia la Declaración de Impacto Ambiental favorable del Proyecto de recuperación y adecuación ambiental de la Bahía de Portmán (BOE, 2011), que incluía el dragado para acercar la línea de rompiente unos 250 m. a la línea de costa de 1957 (Martínez y Pérez-Sirvent, 2013). En septiembre de 2011 se publica en el BOE y en el Diario Oficial de la Unión Europea la licitación para la adjudicación del Proyecto. Sin embargo, tras las elecciones generales en noviembre 2011, el Ministerio de Agricultura, Alimentación y Medio Ambiente (MAGRAMA) anuncia el desistimiento del procedimiento de contratación (BOE, 2012), de licitación de las obras de recuperación ambiental de la Bahía de Portmán. En julio de 2013, se anuncia el inicio de un expediente para declarar los residuos de la Bahía de Portmán como recurso minero a instancia de las mercantiles Aria Internacional GmbH y Acciona Infraestructuras SA (BORM, 2013). Finalmente, se produjo el encausamiento del administrador único de ARIA y el Ministerio declaró desierto el concurso de adjudicación para la explotación minera.

En definitiva, las expectativas de un proyecto de recuperación y adecuación ambiental de la bahía de Portmán promovido por el Ministerio de Medio Ambiente, con el consenso de las administraciones implicadas y todos los colectivos vecinales y ecologistas, desde finales de 2011 se encuentra paralizado. A esta situación se une la propuesta de la Autoridad Portuaria de Cartagena para la ubicación de una nueva terminal de contenedores en la cala de El Gorguel, localizada en las proximidades de la bahía de Portmán, con una superficie de ocupación marina de unas 340 ha., más una Zona de Actividades Logísticas de otras 200 ha., lo que conlleva, más si cabe, un nuevo motivo de incertidumbre a una comarca que demanda desde hace décadas la restauración ambiental de la bahía de Portmán y su entorno (FSM, 2009).

Desde un punto de vista socioeconómico, la regeneración de la bahía puede suponer el comienzo de un cambio de rumbo en los enfoques habituales de desarrollo turístico de áreas costeras. La necesidad de un modelo alternativo en este sector queda patente por 
el momento de estancamiento en el que se encuentra el turismo de sol y playa (García Sánchez y Alburquerque García, 2003; Amat Montesinos, 2011). Siguiendo los patrones convencionales de desarrollo, el turismo se puede utilizar como un instrumento para la construcción de nuevos espacios de crecimiento territorial (Benseny, 2006), pero las presiones ejercidas por un aumento de la población estacional en la costa, ponen en peligro la sustentabilidad ambiental del entorno (Espinosa y Rodríguez, 2015). El espacio litoral mediterráneo ha experimentado una degradación ambiental como consecuencia de la implantación turística, por lo que el cuidado de estos paisajes requiere un conocimiento de su morfología y procesos naturales, basado en la fragilidad y alta vulnerabilidad de sus ecosistemas, así como en una cuidadosa planificación y gestión sostenible (Vera Rebollo et al., 1997; López Bermúdez, 2013).

Son incuestionables los beneficios económicos que puede generar la actividad turística, y su papel de motor de desarrollo económico en el sistema productivo internacional (Brida et al., 2008; Eugenio-Martin y Campos-Soria, 2014), pero su ejercicio improvisado y exacerbado, ha contribuido en muchas ocasiones al deterioro irreversible de los recursos naturales, valor principal de la propia actividad turística. Esto supone una amenaza, no sólo en la práctica turística futura sino para el propio bienestar de las comunidades receptoras. Ello obliga a replantear nuevos enfoques, y modelos conceptuales y operativos, que sirvan para encontrar perspectivas de sustentabilidad más acordes con los desarrollos turísticos, que mejoren la presión socio-ambiental ejercida por los esquemas más tradicionales (González y León, 1996; Morillo-Moreno, 2001).

Además, es esencial establecer otros elementos que favorezcan el bienestar del municipio, contemplando el territorio como una parte principal del progreso socio-económico y no solamente como un marco de actividades económicas (Sánchez-Silvera et al., 2012).

Uno de los principales objetivos de este trabajo es analizar el posicionamiento de los distintos agentes sociales ante las propuestas previas y actuales presentadas para el desarrollo de actuaciones de regeneración de la bahía de Portmán y su entorno. Así mismo, se pretende elaborar y analizar una matriz D.A.F.O. que permita determinar las posibilidades de actuación y las directrices que deben seguir los procesos de planificación estratégica previstos en el área de estudio, con el fin de elaborar un modelo real de desarrollo turístico sostenible.

\section{2. ÁREA DE ESTUDIO Y METODOLOGÍA}

\subsection{Localización}

La bahía de Portmán está situada en el SE de la Península Ibérica, en el extremo suroriental de la Cordillera Bética que la encierra y aísla por el N, el E y el O (Figura 1). Forma parte de la Sierra Minera de Cartagena, cadena montañosa litoral con relieves que no superan los $450 \mathrm{~m}$ de altitud, y que se extienden de $\mathrm{O}$ a $\mathrm{E}$ desde la ciudad de Cartagena hasta Cabo de Palos (Manteca y Ovejero, 1992). 


\section{Figura 1 \\ LOCALIZACIÓN GEOGRÁFICA DE LA ZONA DE ESTUDIO}

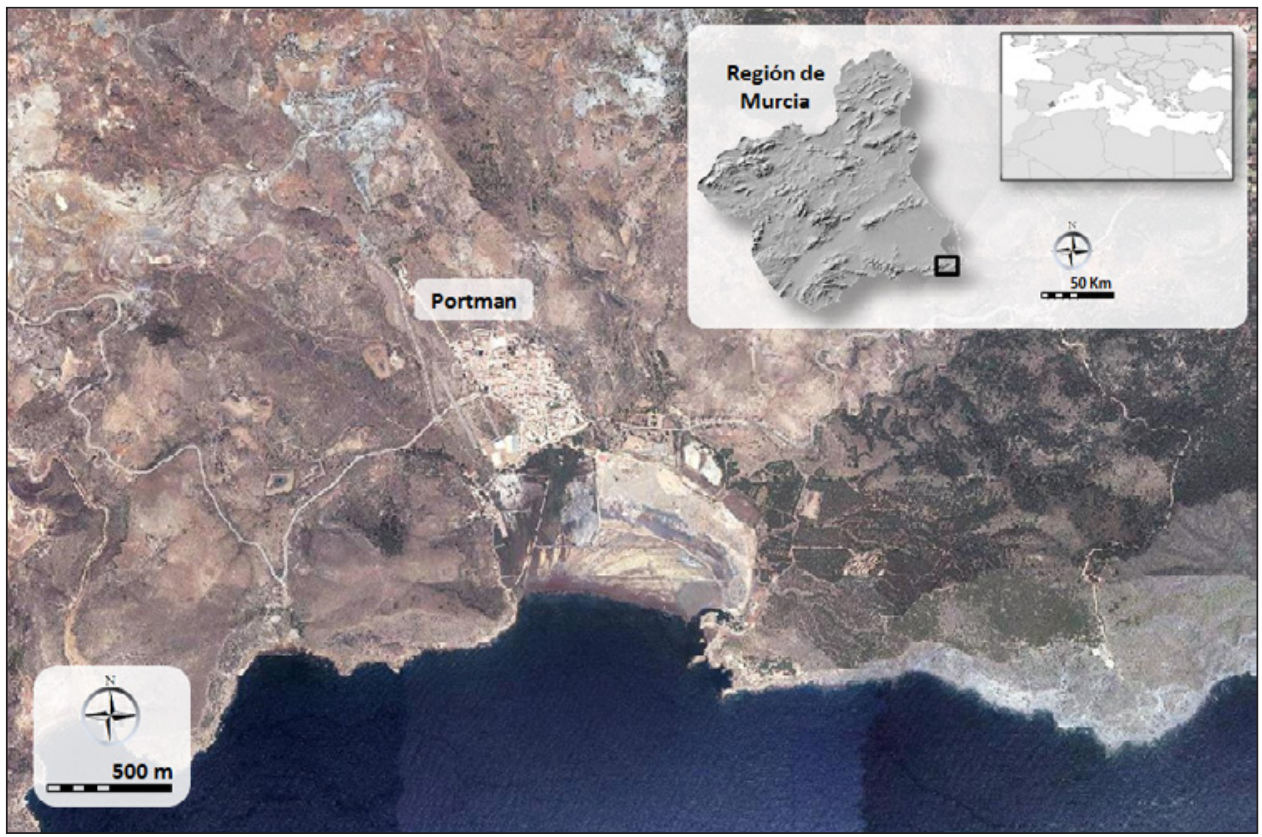

Fuente: elaboración propia.

\subsection{Material y métodos}

\subsubsection{Agentes implicados}

La evaluación de las posiciones de los distintos agentes sociales examinados, respecto al desarrollo y regeneración de la bahía, se ha realizado mediante análisis de observación participante (Ander-Egg, 1987; García Ferrando et al., 1989; Kawulich, 2005; Hennink et al., 2010), del cual se extrae una tabla de cuantificación de las principales posturas establecidas. Para realizar el análisis de las diferentes actuaciones y soluciones propuestas para la regeneración y el desarrollo turístico de la bahía de Portmán y Sierra Minera, se definen a continuación los principales agentes sociales implicados en la historia reciente de Portmán para así poder entender los distintos posicionamientos que defienden:

- La Confederación Regional de Organizaciones Empresariales de Murcia (CROEM) y la Confederación de Organizaciones Empresariales de Cartagena (COEC) apoyan la recuperación del espacio afectado por las actividades mineras para potenciar un desarrollo urbano-turístico importante en la comarca. Coinciden con las propuestas de la mercantil Portmán Golf S.A., que se presenta como única gran empresa implicada, ostentando una posición de monopolio en el mercado del suelo de esta zona, 
con más del $80 \%$ de los terrenos del núcleo urbano de Portmán y sus alrededores, así como gran parte del terreno de la Sierra Minera de Cartagena-La Unión, desde que la empresa Peñarroya-España le transfirió sus propiedades.

- La Fundación Sierra Minera, organización de carácter participativo que aglutina el tejido asociativo de los pueblos de la Sierra Minera de Cartagena-La Unión, integra diversos colectivos vecinales, cuyo ámbito de actuación es la comarca, incluyendo asociaciones de vecinos, con especial dedicación de la asociación Liga de Vecinos de Portmán. La Fundación Sierra Minera es miembro de Europamines, la Red del Patrimonio Minero Europeo.

- La presencia de organizaciones científicas ha sido notable. De manera directa, el Grupo de Investigación en Recuperación de Suelos Contaminados de la Universidad de Murcia (CSOILS) ha desarrollado importantes trabajos científicos para el tratamiento de los estériles más contaminados de la bahía. También es destacable la participación de diversos componentes del Dpto. de Ecología e Hidrología y del Dpto. de Sociología y Trabajo Social de la Universidad de Murcia. Así mismo, es destacable la participación del Área de Geodinámica de la Universidad Politécnica de Cartagena (UPCT); las áreas de Arqueología y de Historia Moderna y Contemporánea de la Universidad de Murcia; el Dpto. de Ciencias del Mar y Biología Marina de la Universidad de Alicante (UA). El Instituto Español de Oceanografía, el Centro de Estudios y Experimentación de Obras Públicas (CEDEX) y el Instituto Geológico y Minero de España (IGME) también han venido colaborando activamente en el estudio y seguimiento de las propuestas para tratar la problemática ambiental de la zona.

- Los grupos ecologistas han desempeñado desde el principio un importante papel de denuncia en la degradación ambiental de la comarca. Desde mediados de la década de 1970, varias personas de la zona vinculadas al Grupo Ecologista Mediterráneo realizan denuncias contra el vertido de esos estériles mineros sin depurar. Otras agrupaciones como la Asociación de Naturalistas del Sureste (ANSE) y Ecologistas en Acción, a través de acciones de protesta y movilizaciones participan en estudios, propuestas de actuación y alegaciones; mantienen una posición destacada en pro de la adecuación ambiental de la sierra y la Bahía de Portmán. A mediados de la década de 1980, con el cierre simbólico de las tuberías de vertido al mar por la organización "GreenpeaceEspaña” y su repercusión en los medios de comunicación, se inicia una nueva etapa en las actuaciones de lucha contra los vertidos de estériles de minería en Portmán.

- Por la particularidad del caso, la implicación de la Administración pública se va a producir en todos los rangos de competencia.

- La Administración local con mayor competencia es el Ayuntamiento de La Unión, su incidencia sobre el modelo de desarrollo en la zona es básica mediante la gestión de la planificación urbanística a través de los distintos sistemas de planeamiento y ordenación municipal.

- La Administración Autonómica elabora las Directrices de Ordenación y Protección del Litoral de Murcia, y tiene las competencias sobre la Sierra Minera y sobre las aguas interiores del litoral de la Comunidad Autónoma de la Región de Murcia (CARM), excepto el Puerto de Cartagena y sus tres dársenas: la de Cartagena, la de Escombreras y la de Portmán. 
- A partir del momento en que la bahía dejó de ser considerada aguas interiores para ser reconocida como la $3^{\text {a }}$ dársena del Puerto de Cartagena, la competencia de la misma paso a ser de la Administración Central. Las propuestas de recuperación fueron, inicialmente, año 1995, desde la Secretaría de Estado de Medio Ambiente y Vivienda, en el Ministerio de Obras Públicas, Transportes y Medio Ambiente (MOPTMA). El proyecto del Ministerio de Medio Ambiente fue sometido a información pública en 2007 y la declaración de impacto ambiental favorable del Ministerio de Medio Ambiente, Medio Rural y Marino, en 2011. Y finalmente, la Unión Europea a través de posibles fuentes de financiación y de supervisión en la ejecución; además de las garantías medioambientales requeridas para las distintas actuaciones.

\subsubsection{Análisis D.A.F.O.}

A través de un análisis D.A.F.O. (Weihrich, 1982; Helms y Nixon, 2010) se pretende investigar los procesos de planificación estratégica previstos en el área de estudio, útiles para la aportación de información necesaria, y válidos para la ejecución de acciones y medidas correctivas que se pueden incluir en el desarrollo de estrategias de mejora.

Es primordial determinar cuáles son las fortalezas, oportunidades, amenazas y debilidades que mayor impacto puedan causar sobre el cumplimiento de las acciones planificadas en la bahía de Portmán. Para la caracterización de los elementos D.A.F.O. se han valorado factores sociales, económicos y políticos que pueden intervenir de manera directa en las acciones planificadas.

La obtención de una matriz de diagnóstico permite analizar los factores externos, expresados a través de las oportunidades y amenazas; y los factores internos, mediante las fortalezas y las debilidades, facilitando la elaboración de posibles escenarios que sirven para evaluar la intensidad de interacción aplicando un valor de impacto entre los factores seleccionados.

Para poder cuantificar este análisis, se ha elaborado una matriz de confrontación que establece el grado de influencia de las externalidades sobre los factores internos a través de las relaciones existentes entre las oportunidades y amenazas respecto a las fortalezas y debilidades. Los valores utilizados expresan una influencia nula en la confrontación entre pares (0), baja (1), media (2) y alta (3). De esta forma se obtienen una suma de cuatro interacciones, que permiten diseñar estrategias adecuadas para afrontar posibles escenarios futuros. Los valores elevados para cada una de estas relaciones significan:

- Fortalezas y Oportunidades (F-O), potenciación de las Fortalezas. Se pueden plantear estrategias de desarrollo, tratando de obtener el máximo partido a esta situación positiva.

- Fortalezas y Amenazas (F-A), disminución de las Fortalezas. Para evitarlo, se deben plantear estrategias activas, tratando de favorecer las potencialidades del entorno y minimizar las amenazas.

- Debilidades y Oportunidades (D-O), disminuye las Debilidades. Las estrategias a llevar a cabo son de reorientación, en las que se pueden aprovechar situaciones futuras positivas para corregir carencias. 
- Debilidades y Amenazas (D-A), se potencia las Debilidades. Se deben emplear las estrategias más conservadoras, que minimicen la posibilidad de que las amenazas externas acentúen los puntos débiles.

\section{RESULTADOS Y DISCUSIÓN}

\subsection{Posicionamiento de los agentes sociales respecto a las propuestas de desarrollo}

Para sintetizar el posicionamiento de los agentes sociales respecto a la regeneración de la bahía de Portmán y el desarrollo de su entorno se presenta una descripción de los objetivos y acciones que han desempeñado a largo de todo el proceso (Tabla 1). A través de la observación participante y diversos proyectos de investigación, se ha explorado este posicionamiento ante los diferentes ejes de progresión socioeconómica y ambiental que puede adoptar el futuro desarrollo de Portmán y su entorno, del cual se puede extraer algunos patrones comunes (Tabla 2).

Las posturas de los diferentes actores aparecen claras en la mayoría de las actividades que se contemplan. El eje de consenso ante las distintas actividades es la rehabilitación/ recuperación de usos de la bahía de Portmán, en la que todos los actores considerados tienen una posición favorable. Claramente a favor están los colectivos vecinales, grupos ecologistas, organizaciones científicas y también la posición de la Administración Central, sobre todo en el periodo 2004-2011. Más tenues son las posiciones de los ayuntamientos implicados y la Comunidad Autónoma de la Región de Murcia, favorables a la recuperación pero vinculándola en muchos casos, función del equipo de gobierno correspondiente, a desarrollos urbano-turísticos y náutico-deportivos. Menos perceptible es la actitud de los grandes propietarios del territorio, que no se han opuesto pero no han mostrado empeño ni apoyo para una adecuada restauración ambiental.

Por el contrario, las actividades que menos consenso suscitan son la creación de la dársena deportiva en la bahía de Portmán y el establecimiento del puerto de contenedores en la Cala de El Gorguel, con una posición de apoyo clara y decidida por parte de todos los niveles en las administraciones implicadas, con la excepción del ayuntamiento de La Unión.

Desde el punto de vista de los actores, el análisis en las posiciones más definidas se dan en los grupos ecologistas, con el apoyo de manera activa a las actividades de conservación y de recuperación, tanto del patrimonio natural, biótico y abiótico, como minero-cultural. Manifiestan rechazo ante la imposición del modelo de desarrollo vinculado a las grandes infraestructuras portuarias: deportiva y de contenedores. Se posicionan en la recuperación del puerto tradicional, y dan apoyo a un desarrollo urbanístico limitado y respetuoso con los valores culturales y naturales del entorno de la bahía de Portmán.

Los grandes propietarios, la empresa Portmán Golf S.A., apoyan la recuperación de usos de la bahía de Portmán en la medida que sería el motor para favorecer un desarrollo urbano-turístico beneficioso a sus intereses. Su enfoque cambia respecto a la restauración de la sierra y la conservación y recuperación del patrimonio natural y cultural, apostando decididamente por el desarrollo urbanístico y por el establecimiento de la dársena deportiva y el puerto de contenedores en El Gorguel. 


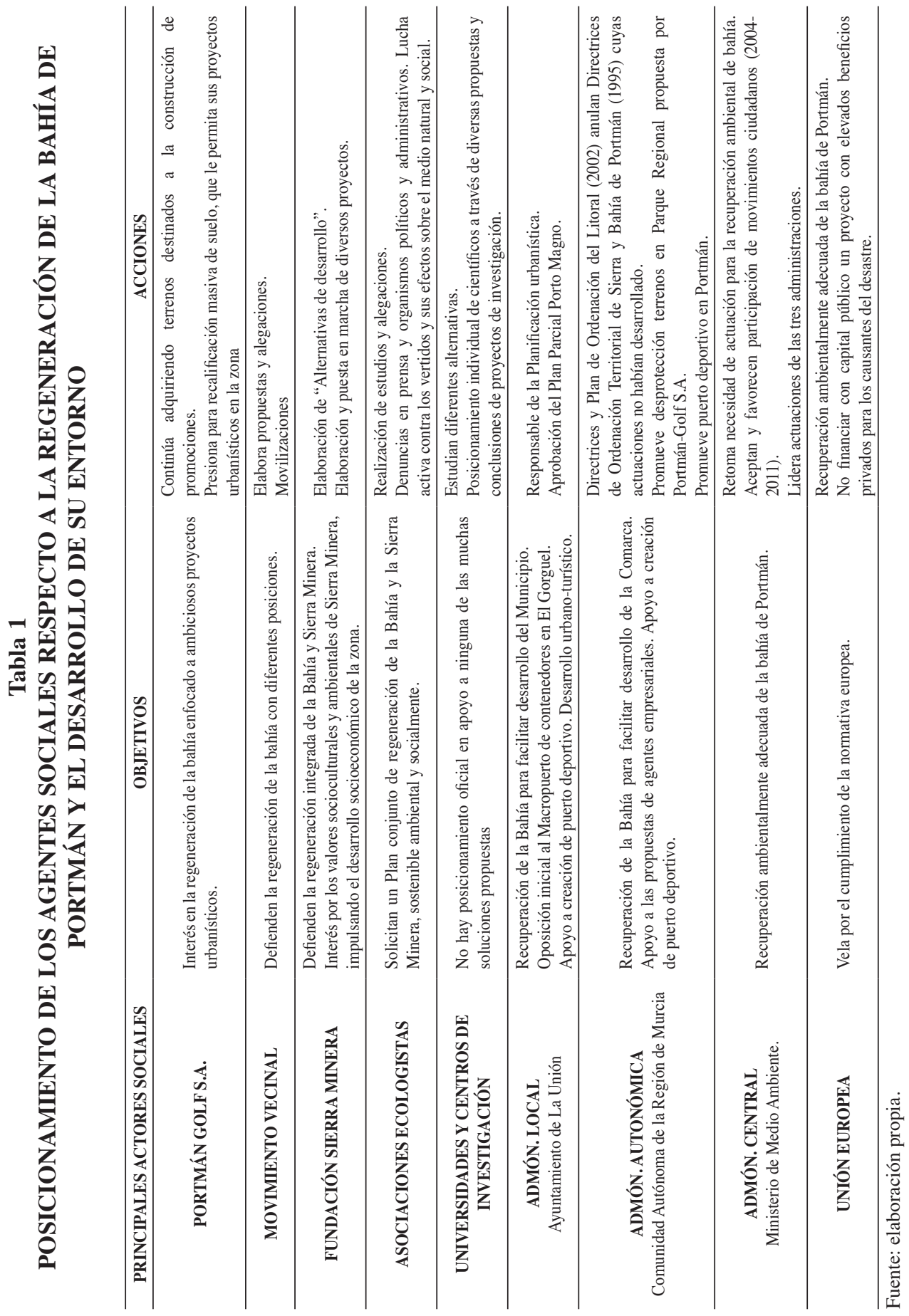




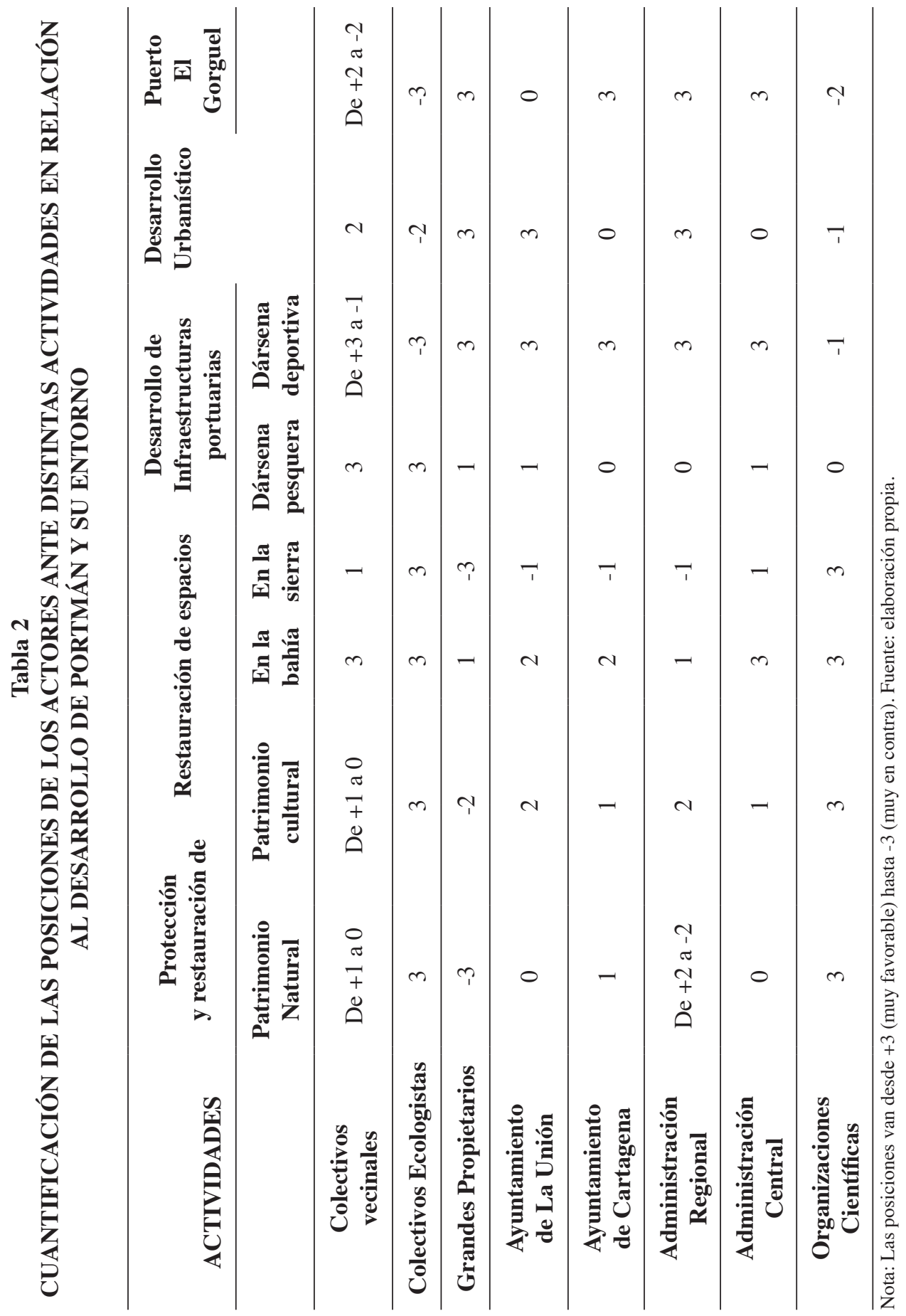


Más heterogéneas son las posiciones que presentan los colectivos vecinales, lo que muestra las diversas posturas e intereses en estas agrupaciones. Sólo hay un cierto consenso hacia las tareas de recuperación de la bahía y el desarrollo de actuaciones que mejoren la actividad turística. En el resto, no existe una oposición patente pero tampoco unas posturas decididas de apoyo a su conservación y recuperación. En cuanto al desarrollo turístico, se pone de manifiesto la oposición de todos los colectivos ambientales a un modelo de desarrollo urbanístico basado en un crecimiento exponencial de este sector y la falta de apoyo de algunos de los colectivos vecinales.

De este análisis queda evidente la existencia de dos grandes conjuntos; por una parte los que deciden primar una mayor integración ecológica y cultural del proyecto de regeneración de la bahía; y por otra, las iniciativas o posicionamientos dirigidos a un mayor desarrollo turístico-económico a corto plazo. Con estos ingredientes, cada actor social percibe de una manera diferente el futuro desarrollo de la zona. En la práctica, puede entenderse como la materialización de dos modelos de desarrollo parcialmente excluyentes: uno más sostenible y respetuoso con los valores naturales y culturales de la zona, que podría tener un perfil pionero y singular en la costa mediterránea de la Región de Murcia; y otro que puede entenderse como la simple proyección tendencial del crecimiento económico que se ha dado en el litoral murciano durante las últimas décadas y que responde fundamentalmente al statu quo del poder económico vigente.

\subsection{Diagnóstico D.A.F.O.}

Las externalidades de la minería han sido un tema de debate continuado en la historia de este sector, en las que buena parte de las zonas con un pasado extractivo son en la actualidad zonas deprimidas o de nula industrialización (Manteca et al., 2005). En el caso de Portmán, a pesar de su situación geográfica excepcional (junto a un centro turístico de primer nivel como es el complejo de Atamaría-La Manga Club) y a los valores naturales de su entorno, las externalidades socioeconómicas y ambientales heredadas de la actividad minera acentúan aún más la vulnerabilidad de esta población y sus alrededores en cuanto a las oportunidades para un desarrollo sostenible y coherente.

Para su análisis, se ha elaborado un catálogo de los elementos más significativos en el planeamiento estratégico de la zona de estudio (Tabla 3), y que han servido de entrada para la obtención de una matriz de confrontación DAFO (Tabla 4), que recoge las principales debilidades, amenazas, fortalezas y oportunidades que caracterizan la zona de estudio.

Como se observa en el análisis de los resultados de la matriz DAFO (Figura 2), el cuadrante que muestra la mayor puntuación es la referente a la situación F-O (Fortalezas ante Oportunidades). Esta posición nos muestra una evaluación muy positiva de las fortalezas de la comarca ante las posibles oportunidades externas, que pueden fomentar procesos de retroalimentación ventajosos. Entre las fortalezas hay que destacar la calidad y singularidad del entorno, así como la presencia de hábitats muy raros y prioritarios correspondientes a Matorrales arborescentes de Ziziphus (hábitat 5220*), caracterizado por Periploca angustifolia y Maytenus senegalensis subsp. europea, y Bosques de Tetraclinis articulata (hábitat 9570*). Su excelente grado de conservación, unido a la escasa extensión a escala nacional de estos hábitats, determinaría la inclusión en la Red Natura 
Tabla 3

MATRIZ D.A.F.O.

\section{DEBILIDADES}

AMENAZAS

D1. Monopolio de la propiedad del suelo por Portmán Golf S.A.

D2. EDAR antigua y de limitada capacidad.

D3. Inexistencia de oferta de alojamientos hosteleros.

D4. Escasa disponibilidad de suelo apto para ser urbanizado

D5. Escasa oferta de turismo complementario, deportivo, cultural, etc.

D6. Bajo atractivo para turismo de sol y playa por la percepción de contaminación.

A1. Crisis económico-financiera y social a nivel global.

A2. Posible construcción de superpuerto de contenedores en El Gorguel.

A3. Olores desde la planta de tratamiento de RSU en El Gorguel

A4. Posible instalación en la zona de Cruz Chiquita, de un depósito de residuos de suelos contaminados.

A5. Proximidad a la refinería de petróleo en Escombreras, recientemente ampliada y al amplio complejo petroquímico, terminal de gas natural, centrales de producción de energía eléctrica.

A6. Pendiente de adjudicar el Proyecto de regeneración y adecuación ambiental de la bahía de Portmán licitado en septiembre de 2011, tras el desistimiento por el MAGRAMA en 2012.

\section{FORTALEZAS} OPORTUNIDADES

F1. Amplia oferta de viviendas disponibles.

F2. Entorno de alta calidad paisajístico natural con la existencia de hábitats muy raros y prioritarios.

F3. Paisaje Minero incluido en el Plan de Patrimonio Industrial de 2011 e incoado el procedimiento de declaración de BIC

F4. Espacio turístico no saturado.

F5. Proximidad a importantes polos de atracción turística.

O1. Inclusión de propuestas de desarrollo adecuadas a las condiciones de singularidad de la zona, en el próximo PGMO del municipio de La Unión.

O2. Plan para solicitar a la UNESCO la inclusión de la zona de Cabo de Palos a Cabo Tiñoso como Reserva de la Biosfera.

O3. Posible declaración de Calblanque, Monte de las Cenizas y Peña del Águila como Parque Nacional Marítimo Terrestre. La existencia de hábitats muy raros y prioritarios podría posibilitar inversión europea en relación a su inclusión en la Red Natura 2000.

F6. Disponibilidad de espacio próximo al mar para instalaciones de desarrollo turístico.

O4. Posible creación de un centro de investigación marina para la CARM en la antigua batería C-8 "La Chapa" junto al faro de Portmán.

O5. Inclusión de los espacios de alto valor geológico, biológico, arqueológico, etnográfico en el Parque Minero de La Unión.

O6. Proyecto de puerto deportivo, incluyendo la recuperación y ampliación del puerto pesquero tradicional.

Fuente: elaboración propia. 


\section{Tabla 4 \\ MATRIZ DE CONFRONTACIÓN D.A.F.O}

Factores externos

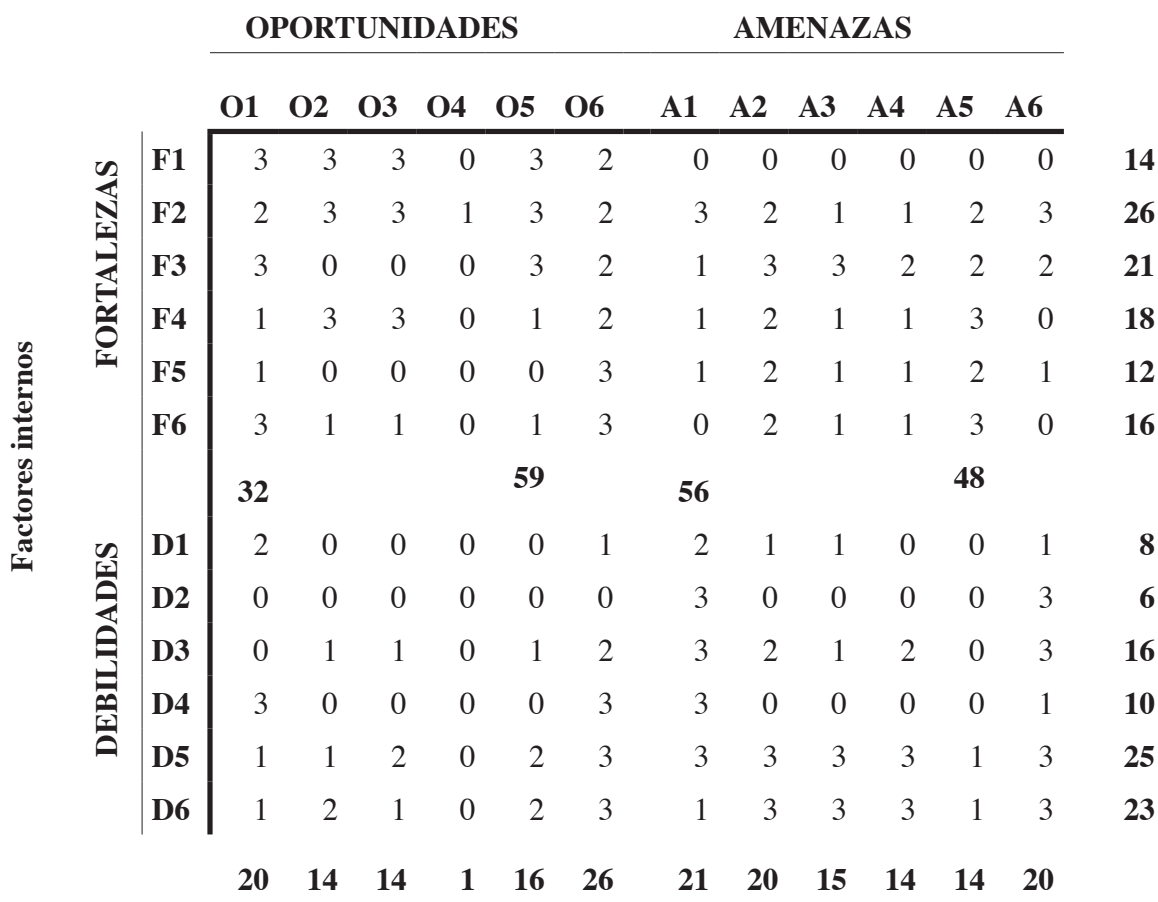

Nota: Nivel de impacto nulo (0); bajo (1); medio (2), alto (3). Fuente: elaboración propia.

2000 de estas zonas, de acuerdo con la Directiva Habitat (D 92/43/CEE, de 21 de mayo de 1992), representando una oportunidad para poder recibir inversión europea en relación con la conservación de la biodiversidad. Los valores naturales más destacables del entorno de la bahía de Portmán incluyen: el Parque Regional de Calblanque, Monte de las Cenizas y Peña del Águila; el paisaje Protegido de Espacios Abiertos e Islas del Mar Menor (que incluye en el área de influencia de Portmán, los parajes de El Sabinar, San Ginés y Las Salinas de Marchamalo); la reserva Marina de Cabo Palos-Islas Hormigas; el Lugar de Importancia Comunitaria (LIC) y Zona de Especial Protección para las Aves de Sierra de Fausilla (incluyendo El Gorguel); los espacios sumergidos LIC Franja Litoral Sumergida y LIC Medio Marino; y Lugar de Interés Geológico (LIG) de Sierra Minera.

En cuanto a los valores culturales del entorno, cabe destacar el catálogo de Bienes Inmuebles de la Sierra Minera y las Cartas Arqueológicas de los municipios de La Unión y Cartagena, realizado entre los años 1992 a 1997. En el año 2006 se procede a una revisión y re-catalogación del mismo, recogiendo un total de 467 elementos. Entre 2004 y 2006 se realizó el Plan Director del Paisaje Industrial de la Sierra Minera de Cartagena-La Unión, 
en el que se recogía una programación de intervenciones y propuesta de uso en las que se incluyeron el túnel José Maestre y la Carretera del 33 de La Unión a Portmán. En septiembre de 2006, se inicia el expediente de declaración BIC-Sitio Histórico la Sierra Minera Cartagena-La Unión, cuya parte más extensa, engloba Portmán y sus alrededores, y en 2009, se declara finalmente BIC la Sierra Minera de Cartagena-La Unión, con categoría de Sitio Histórico. Aunque finalmente fue anulado por sentencia judicial, actualmente está terminándose la adaptación del mismo. Cabe destacar la actuación de la UNESCO que ha incluido la sierra minera de Cartagena-La Unión en la lista indicativa de «Paisajes culturales excepcionales» (BORM, 2009, pp: 19450; BORM, 2012, pp: 44448).

\section{Figura 2}

\section{GRÁFICO RADIAL DE LA MATRIZ DE CONFRONTACIÓN D.A.F.O}

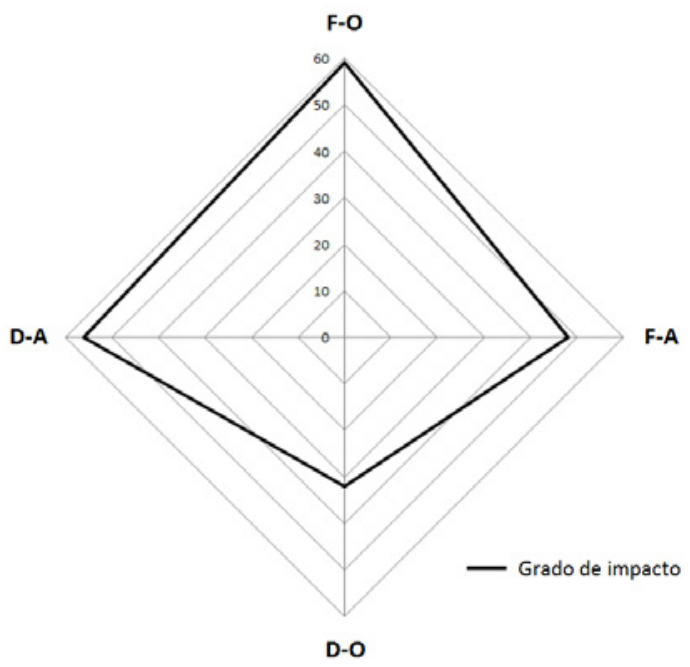

Fuente: elaboración propia.

Uno de los primeros pasos significativos en la recuperación y reutilización del patrimonio industrial de la Sierra Minera fue llevado a cabo por la Fundación Sierra Minera (FSM) con la rehabilitación de la Mina "Las Matildes", estableciendo un centro de interpretación de la minería y la restauración ambiental de su entorno y la puesta en marcha de alternativas de turismo ecocultural. También representaría una oportunidad la inclusión de los espacios de alto valor geológico, biológico, arqueológico, etnográfico de los alrededores de Portmán en el Parque Minero de La Unión, que actualmente llega hasta la vertiente norte de la Sierra Minera. En este sentido, la singularidad minera de Portmán y su entorno permite una oferta única en el contexto de la Región de Murcia. La localización de Portmán al sur del Mar Menor y próxima al complejo de Atamaría-La Manga Club, también fortalece esta propuesta de revalorización, ya que complementa la oferta vacacional, basada en la concentración de conjuntos residenciales. 
En cierta manera, el boom inmobiliario ha facilitado la existencia de un stock de viviendas en urbanizaciones recientes, que puede servir para ampliar la oferta residencial, y aprovechar los mencionados polos de desarrollo turísticos de la zona. La escasa saturación turística termina por apoyar los posibles intentos de promover un desarrollo turístico sostenible manteniendo su patrimonio ambiental y geológico-arqueológico-minero. Por último, existe la disponibilidad de amplios espacios cercanos a la bahía de Portmán para la construcción de instalaciones de apoyo al desarrollo turístico.

Igualmente alta es la evaluación de las D-A (Debilidades ante Amenazas), lo cual nos presenta un escenario transversalmente distinto al cuadrante F-O, en el que las posibles amenazas incrementan las fragilidades existentes. La propiedad del suelo de Portmán y su entorno, se caracteriza por una situación de casi-monopolio de la empresa Portmán Golf S.A, que adquirió de Peñarroya-España, por lo que cualquier iniciativa de desarrollo en este sentido debe ser consensuada con esta entidad.

En general, la baja cualificación de la población desempleada unido a la escasa oferta de empleo en la localidad ha influido en el aumento de los procesos de emigración (CREM, 2016). Otro factor que habría que tener en cuenta en estos procesos es la escasez de transporte público por carretera y la mala calidad de las infraestructuras de comunicaciones. Portmán está calificado como Punto de Especial Aislamiento desde la perspectiva de la atención sanitaria.

El deficitario y anticuado sistema de distribución de agua potable, así como la estación depuradora de aguas residuales (EDAR) y la red de alcantarillado, suponen una notable debilidad en las posibilidades de abastecimiento y depuración de aguas para el desarrollo turístico. Está pendiente la construcción de una nueva EDAR.

La demanda de viviendas de $2^{\text {a }}$ residencia es muy baja, y casi nula la oferta de alojamientos de alquiler, incluso no reglado, con ninguna plaza alojativa turística (actualmente hay un pequeño hotel en construcción). Además, la oferta de turismo complementario (deportivo, cultural, etc.) es prácticamente inexistente, a lo que ha de añadirse la dificultad de acceso a centros y espacios BIC por las restricciones de la propiedad. En cuanto al tradicional turismo de sol y playa, el atractivo turístico de la bahía es relativamente bajo, por las condiciones de la propia playa y alrededores, por la percepción de riesgo para la salud pública por contaminación de arenas y aguas de baño.

El análisis de las limitaciones para el desarrollo desde las perspectivas ambientales, patrimoniales y territoriales derivadas de la ordenación del suelo de la cuenca visual de la bahía de Portmán, ofrece una serie de condicionantes. De las casi mil hectáreas que incluye la zona del área visual desde la bahía, y en relación a posibles desarrollos residenciales, cabe tener en cuenta que unas 500 ha. están incluidas dentro de categorías con limitaciones importantes (Dominio Público Marítimo Terrestre y Zona de Servidumbre de Protección; ámbito de los recientes incendios de 2010 y 2011; Protección Geomorfológica por pendientes, establecido en las Directrices de Ordenación y Protección del Litoral de la Región de Murcia; Red Natura 2000). Otras 700 ha. aproximadamente, estarían sujetas a condicionantes principales para los desarrollos urbanísticos derivados de la necesidad de eliminación de los riesgos mineros y de contaminación de suelos, además de los derivados del mantenimiento de los valores naturales del entorno. Cabe destacar otra serie de condicionantes adicionales a los anteriores, derivados de la protección del patrimonio natural y 
del patrimonio arqueológico, que suponen unas 880 ha. Estos condicionantes adicionales -incluyendo las afectadas por factores limitantes- afectan a más del 90\% del entorno de la bahía de Portmán en su cuenca visual. Por lo que se puede concluir que la disponibilidad de suelo apto para ser urbanizado en las proximidades al actual núcleo urbano es escasa.

En el cuadrante F-A (Fortalezas ante Amenazas) se muestra un valor relativamente alto, lo que significa que las fortalezas de la comarca no son lo suficientemente potentes para afrontar las amenazas externas. El presente contexto de crisis económico-financiera y social a nivel global dificulta las inversiones -incluso las ya comprometidas- para la restauración ambiental de la bahía de Portmán; así como para la construcción y explotación en régimen de concesión a iniciativa privada de la marina deportiva prevista para realizar tras la recuperación de la bahía de Portmán.

La posible construcción de un "superpuerto" de contenedores en El Gorguel, a unos 1.000 m. de la bahía de Portmán, amenazaría no sólo los valores ambientales del entorno de la bahía de El Gorguel, la Sierra de la Fausilla, y la protección del patrimonio minero de El Gorguel, sino también las posibilidades de desarrollo turístico y residencial del entorno.

Otro elemento a tener en cuenta es la prevista instalación en las inmediaciones de la mina Cruz Chiquita de un depósito de residuos de suelos contaminados de las antiguas instalaciones industriales de Potasas y Derivados y de Española del Zinc, que podría perjudicar la atracción de inversores o iniciativas de desarrollo en el sentido anteriormente citado. A ello, hay que añadir la cercanía de la planta de tratamiento de Residuos Sólidos Urbanos (RSU) en El Gorguel y de la refinería de petróleo en Escombreras que supone contaminación atmosférica hacia Portmán cuando soplan vientos de poniente; además del riesgo por posible accidente en el polo petroquímico de Escombreras. También es significativa la contaminación atmosférica y de suelos debida a la existencia de pantanos de estériles mineros en las proximidades de la localidad (Belmonte et al., 2010).

Por último, la grave amenaza que supone para todo el proceso de recuperación de la bahía de Portmán y su entorno el desistimiento por el MAGRAMA del procedimiento de contratación del Proyecto de regeneración y adecuación ambiental de la bahía de Portmán (BOE, 2012); lo que ha supuesto importantes retrasos al tener que volver a convocar los concursos de adjudicación de obras y hacer mucho más difícil poder recibir ayudas de fondos europeos para el Proyecto.

Finalmente, la posición D-O (Debilidades ante Oportunidades) es la que menor puntuación obtiene, expresando que las debilidades analizadas pueden ser abordadas por posibles escenarios favorables, lo que subraya la importancia de una adecuada planificación y gestión ante las deficiencias estructurales de la comarca. A través de propuestas de desarrollo adecuadas en el Plan General Municipal de Ordenación del municipio de La Unión, se puede llevar a cabo la recuperación y la rehabilitación de los restos mineros y la puesta en valor de los espacios de mayor valor ambiental de Portmán y la Sierra Minera de Cartagena-La Unión. En este sentido, uno de los mayores impulsos estaría en la posible declaración de Calblanque, Monte de las Cenizas y Peña del Águila como Parque Nacional Marítimo Terrestre.

En el Informe preliminar sobre la viabilidad de esta declaración iniciado en 2005 desde la Dirección General del Medio Natural de la CARM, se analizaron y cumplimentaron los 
requisitos necesarios exigidos por la UNESCO para que dicho espacio natural pudiera ser declarado Reserva de la Biosfera. Con una posible ampliación de este segmento costero hasta Cabo Tiñoso-Peñas Blancas, constituiría la propuesta de Reserva de la Biosfera de las Sierras Costeras y Sistemas Marinos de Cartagena-La Unión (CARM, 2009; BañosGonzalez et al., 2012). Una magnifica marca para un nuevo modelo de desarrollo más sostenible, atractivo para el turismo etnográfico y de naturaleza, y como oferta complementaria a las zonas turísticas cercanas de La Manga y el Mar Menor, cuyo modelo de desarrollo ha sido, casi exclusivamente, turismo de sol y playa. Todo este complejo de áreas protegidas terrestres y marinas y sus correspondientes corredores ambientales podría representar el concepto matriz para estructurar un nuevo diseño territorial en este segmento costero de la costa murciana. En este nuevo diseño, la conservación y restauración ambiental y del patrimonio cultural resultarían ejes fundamentales para un desarrollo sustentable, basado en los valores del patrimonio natural y geológico-minero de la zona, diferente a los modelos más convencionales de turismo.

Por otro lado, Portmán puede constituirse como un referente en el tratamiento de residuos contaminados, y a partir de la futura regeneración y adecuación ambiental de la bahía se podría crear, aprovechando las experiencias en el laboratorio instalado por TRAGSA en la propia Bahía, un Centro de Investigación Especializado en la restauración de zonas degradadas por explotaciones mineras. En esta línea la antigua batería C-8 "La Chapa", ubicada junto al faro de Portmán y cuyas instalaciones fueron adquiridas y rehabilitadas por el Ministerio de Medio Ambiente, representa una oportunidad para la creación de un Centro de Investigación Marina de la Comunidad Autónoma de la Región de Murcia, lo que supondría un importante complemento científico al propuesto Parque Nacional Marítimo Terrestre de Calblanque, Monte de las Cenizas y Peña del Águila. Incluso la recuperación de uso de las instalaciones en la antigua batería de costa C-9 "Las Cenizas" como centro de investigación y vigilancia ambiental, además de musealizar para su visita las impresionantes piezas de artillería e instalaciones complementarias, allí presentes. Estos centros podrían complementarse e integrarse con un área de recepción e interpretación de la bahía, mediante la puesta en valor de los atractivos de la zona y la exposición de las fases de transformación hasta su proceso de restauración.

\section{CONCLUSIONES}

Tras más de 26 años del cierre de los vertidos de estériles al Mar Mediterráneo y cerca de sesenta años desde el inicio del último ciclo económico de la actividad minera, se puede ver un horizonte más esperanzador, aunque todavía con luces y sombras, para la bahía de Portmán y su entorno ecogeográfico.

En el último periodo, las señales positivas de la Administración Central a través de sus estudios técnicos y sus compromisos políticos, se modifican tras el cambio de gobierno de noviembre de 2011, alineándose a la posición de las administraciones locales y autonómica que, aunque con ciertos matices, han mantenido una apuesta instrumental por la regeneración de la bahía como simple motor de reactivación de la actividad económica comarcal. Con ello, se pretende convertir la idea de restauración ambiental original en mera mercancía urbanística, desde una mirada cortoplacista y conservadora en cuanto a 
las expectativas creadas. La Administración Central no parece mantener el mismo compromiso y sensibilidad con la labor de restauración de una bahía, que supone una de las deudas ecológicas y sociales más relevantes de nuestro país.

Los resultados del análisis D.A.F.O. presentados en este trabajo, han puesto de manifiesto las potencialidades para un turismo ambiental y cultural de estos extraordinarios parajes. Sin embargo, las propias debilidades y amenazas podrían superar esas potencialidades si no se lleva a cabo una gestión cuidadosa y una apuesta clara por un modelo de desarrollo sensible en extremo con el patrimonio natural, paisajístico y cultural.

Un proyecto como el de recuperación y adecuación ambiental de la bahía de Portmán, promovido por el Ministerio de Medio Ambiente, que contaba con la Declaración de Impacto Ambiental favorable y con el apoyo de la UE y el acuerdo de las administraciones implicadas y todos los colectivos vecinales y ecologistas que han venido participando en el proceso, representaba una oportunidad que hubiera favorecido las fortalezas de Portmán y su entorno, e impulsado un modelo de turismo vinculado a procesos de desarrollo local.

Un modelo de turismo ligado a la restauración ambiental y al consenso de los diferentes agentes sociales locales, que asuman el proyecto como propio, constituye un elemento básico para la adaptación del territorio como producto turístico. Y así debe reflejarse en las futuras decisiones.

\section{BIBLIOGRAFÍA}

AMAT MONTESINOS, X. (2011): «La expansión urbanizadora en el interior alicantino. Indicadores e incertidumbres», Urbanismo expansivo: de la utopía a la realidad Comunicaciones XXII Congreso de Geógrafos Españoles. Universidad de Alicante.

ANDER-EGG, E. (1987): Técnicas de Investigación Social México. Editorial Humanitas. BANOS-GONZÁLEZ, I. y BAÑOS PÁEZ, P. (Eds). (2013): Portmán: de El Portus Magnus del Mediterráneo Occidental a la Bahía Aterrada Murcia. EDITUM.

BANOS-GONZÁLEZ, I., BAÑOS PÁEZ, P. y ESTEVE SELMA, M.A. (2012): "Paisajes Mineros, Conflictos Ambientales y Participación Social. Génesis y evolución del problema ecológico de la Bahía de Portmán”. En: Sabater Sánchez, Martínez Solano y González Márquez (Coords). Compromiso por la protección del paisaje, la cohesión social y el desarrollo sostenible en la Sierra Minera de Cartagena. Murcia. Centro de Estudios Económicos y Empresariales de la Universidad de Murcia, pp. 25-105.

BAÑOS PÁEZ, P. (2012): Historia de la degradación de la bahía de Portmán Murcia. EDITUM Universidad de Murcia. Disponible en:

http://revistas.um.es/dtsa/article/view/152751/142171

BENEDICTO, J., MARTÍNEZ-GÓMEZ, C., GUERRERO, J., JORNET, A., y RODRÍGUEZ, C. (2008): «Metal contamination in Portman Bay (Murcia, SE Spain) 15 years after the cessation of mining activities». Ciencias Marinas, 34(3), pp. 389-398.

BENEDICTO, J., MARTÍNEZ-GÓMEZ, C., y RUÍZ-FERNÁNDEZ, J. M. (2013): «La Calidad del Medio Marino en Portmán y su entorno: Evolución y Estado Actual de Conocimiento» En: Banos-González y Baños Páez (Eds). Portmán: de El Portus Magnus del Mediterráneo Occidental a la Bahía Aterrada. EDITUM, pp. 345-392. 
BELMONTE, F., ROMERO, A. y MORENO, J. (2010): «Contaminación ambiental por estériles mineros en un espacio turístico en desarrollo, la sierra minera de Cartagena-La Unión (Sureste de España)» Cuadernos de Turismo, (25), pp. 11-24.

BENSENY, G. (2006): «El espacio turístico litoral» Aportes y Transferencias, 10, pp. 102-122.

BOE [Boletín Oficial del Estado] (2011): Resolución de 10 de febrero de 2011, de la Secretaría de Estado de Cambio Climático, por la que se formula declaración de impacto ambiental del proyecto Regeneración y adecuación ambiental de la Bahía de Portmán, término municipal de La Unión, Murcia. (BOE nº 45 de 22/02/2011) pp. 20530-20560.

BOE [Boletín Oficial del Estado] (2012). Anuncio de la Dirección General de Sostenibilidad de la Costa y del Mar por el que se publica el desistimiento del procedimiento de contratación "Proyecto de regeneración y adecuación ambiental de la bahía de Portmán término municipal de La Unión (Murcia)». Expediente 30-1320. (BOE n ${ }^{\circ}$ 265 de 03/11/2012) pp. 50773.

BORM [Boletín Oficial de la Región de Murcia] (2009): Decreto $n^{\circ}$ 93/2009 por el que se declara bien de Interés Cultural con categoría de sitio histórico la Sierra Minera de Cartagena y La Unión. (BORM no 99 de 02/05/2009) pp. 19445-19515.

BORM [Boletín Oficial de la Región de Murcia] (2012): Resolución de 5 de noviembre de 2012 de la Dirección General de Bienes Culturales, por la que se incoa procedimiento de declaración de bien de interés cultural con categoría de sitio histórico, a favor de la Sierra Minera de Cartagena y La Unión (Murcia). (BORM nº 262 de 12/11/2012) pp. 44444-44511.

BORM [Boletín Oficial de la Región de Murcia] (2013): Anuncio de la Dirección General de Industria, Energía y Minas sobre inicio de expediente para la Declaración de Recurso de la Sección B) de la Ley 22/1973, de 21 de julio, de Minas, de los residuos mineros de la Bahía de Portmán, en los términos municipales de La Unión y Cartagena (Murcia). (BORM no 174, de 29 julio 2013) pp. 30783-30784.

BRIDA, J.G., PEREYRA, J.S., SUCH DEVESA, M.J. y ZAPATA AGUIRRE, S (2008): «La contribución del turismo al crecimiento económico» Cuadernos de Turismo, 22, pp. 35-46.

CARM (2009): «Documento Justificativo para la Declaración de Calblanque, Monte de las Cenizas y Peña del Águila y áreas del entorno como Reserva de la Biosfera». Documento técnico. Consejería de Agricultura y Agua. Murcia.

CREM [Centro Regional de Estadística de Murcia] (2016): Padrón de españoles residentes en el extranjero. Disponible en http://www.carm.es/econet/home.html

ESPINOSA, J.A. Y RODRÍGUEZ, F. (2015): «Tendencias y oportunidades de desarrollo turístico en la Costa Tropical de Granada: la visión de los municipios» Cuadernos de turismo, 35, pp. 133-156.

EUGENIO-MARTIN, J.L. y CAMPOS-SORIA, J.A. (2014): «Economic crisis and tourism expenditure cutback decision» Annals of Tourism Research, 44, pp. 53-73.

FSM [FUNDACIÓN SIERRA MINERA] (2009): Informe técnico sobre el impacto de la instalación de una dársena de contenedores en El Gorguel Disponible en http://www. apc.es/archivos/Declaracion\%20EMAS\%202009\%20APC.pdf 
GARCÍA FERRANDO, M.; IBÁÑEZ, J, y ALVIRA, F. (Comp.) (1989): El análisis de la realidad social. Métodos y técnicas de investigación Alianza Universidad. Madrid.

GARCÍA SÁNCHEZ, A. y ALBURQUERQUE GARCÍA, F.J. (2003): «El turismo cultural y el de sol y playa: ¿sustitutivos o complementarios?» Cuadernos de Turismo, 11, pp. 97-105.

GONZÁLEZ, M. y LEÓN C.J. (1996): «Objetivos y Políticas del Desarrollo Turístico Sostenible: Una Metodología Para el Estudio de la Interacción Turismo-Medio Ambiente» Serie de Ensayos y Monografías, 75, pp. 1-26.

HELMS, M.M. y NIXON, J. (2010): «Exploring SWOT analysis-where are we now? A review of academic research from the last decade» Journal of strategy and management, 3(3), pp. 215-251.

HENNINK, M., HUTTER, I., y BAILEY, A. (2010). Qualitative research methods. London: Sage.

KAWULICH, B.B. (2005): «Participant observation as a data collection method» Forum: Qualitative Social Research, 6, pp. 1-32.

LÓPEZ BERMÚDEZ, F. (2013): «Prólogo» En: Banos-González y Baños-Páez (Eds): Portmán: de El Portus Magnus del Mediterráneo Occidental a la Bahía Aterrada, pp. 13-19.

MANTECA, J.I.; PÉREZ DE PERCEVAL VERDE, M.A. y LÓPEZ-MORELL, M.A. (2005): «La industria minera en Murcia durante la época contemporánea» En: Parra Lledó, M. (dir.). Bocamina. Patrimonio minero de la Región de Murcia. Murcia. Ayuntamiento de Murcia, pp. 123-134.

MANTECA, J.I. y OVEJERO, G. (1992). «Los yacimientos Zn, Pb, Ag, Fe del distrito minero de La Unión-Cartagena, Bética Oriental» En: Recursos Minerales de España. CESIC. Col. Textos Universitarios, 15, pp. 1085-1102.

MARTÍNEZ M.J. y PÉREZ-SIRVENT, C. (2013): «Diagnóstico y recuperación de la contaminación del suelo en Portmán-Sierra Minera» En: Banos-González y BañosPáez (Eds): Portmán: de El Portus Magnus del Mediterráneo Occidental a la Bahía Aterrada pp. 313-344.

MARTÍNEZ-FRIAS, J. (1997): «Mine waste pollutes Mediterranean» Nature. 388, pp.120.

MORILLO-MORENO, M. (2001): «Sustentabilidad socio-ambiental de la actividad turística receptora. Reflexiones y alternativas» Economía, 17, pp. 69-107.

SÁNCHEZ-SILVERA, N., BETANCOURT GARCÍA, M.E. y FALCÓN RODRÍGUEZ, M.C. (2012): «Acercamiento teórico al desarrollo local sostenible y su repercusión para el turismo» El Periplo Sustentable, 22, pp. 7-36.

VERA REBOLLO, F., LOPEZ PALOMEQUE, F., MARCHENA GÓMEZ, M. y ANTÓN CLAVE, S. (1997): Análisis territorial del turismo. Una nueva geografía del turismo Editorial Ariel. Barcelona.

WEIHRICH, H. (1982): «The TOWS matrix — a tool for situational analysis» Long range planning, 15(2), pp. 54-66. 